\title{
Obesity-induced gut microbial metabolite promotes liver cancer through senescence secretome
}

\author{
Shin Yoshimoto ${ }^{1,2 *}$, Tze Mun Loo ${ }^{1,2,3}$, Koji Atarashi $^{4,5}$, Hiroaki Kanda $^{6}$, Seidai Sato ${ }^{1,2}$, Seiichi Oyadomari ${ }^{7}$, Yoichiro Iwakura ${ }^{8}$, \\ Kenshiro Oshima ${ }^{9}$, Hidetoshi Morita ${ }^{10}$, Masahisa Hattori ${ }^{9}$, Kenya Honda ${ }^{2,4}$, Yuichi Ishikawa ${ }^{6}$, Eiji Hara ${ }^{1,2}$ \& Naoko Ohtani ${ }^{1,5 *}$
}

\begin{abstract}
Obesity has become more prevalent in most developed countries over the past few decades, and is increasingly recognized as a major risk factor for several common types of cancer ${ }^{1}$. As the worldwide obesity epidemic has shown no signs of abating ${ }^{2}$, better understanding of the mechanisms underlying obesity-associated cancer is urgently needed. Although several events were proposed to be involved in obesity-associated cancer $^{1,3}$, the exact molecular mechanisms that integrate these events have remained largely unclear. Here we show that senescence-associated secretory phenotype (SASP) $)^{4,5}$ has crucial roles in promoting obesity-associated hepatocellular carcinoma (HCC) development in mice. Dietary or genetic obesity induces alterations of gut microbiota, thereby increasing the levels of deoxycholic acid (DCA), a gut bacterial metabolite known to cause DNA damage $^{6}$. The enterohepatic circulation of DCA provokes SASP phenotype in hepatic stellate cells (HSCs) ${ }^{7}$, which in turn secretes various inflammatory and tumour-promoting factors in the liver, thus facilitating HCC development in mice after exposure to chemical carcinogen. Notably, blocking DCA production or reducing gut bacteria efficiently prevents HCC development in obese mice. Similar results were also observed in mice lacking an SASP inducer ${ }^{8}$ or depleted of senescent HSCs, indicating that the DCA-SASP axis in HSCs has key roles in obesity-associated HCC development. Moreover, signs of SASP were also observed in the HSCs in the area of HCC arising in patients with non-alcoholic steatohepatitis ${ }^{3}$, indicating that a similar pathway may contribute to at least certain aspects of obesity-associated HCC development in humans as well. These findings provide valuable new insights into the development of obesity-associated cancer and open up new possibilities for its control.
\end{abstract}

Cellular senescence is a process occurring in normal cells in response to telomere erosion or oncogene activation, acting through checkpoint activation and stable cell-cycle arrest as a barrier to tumorigenesis ${ }^{9,10}$. Recent studies, however, reveal that senescent cells also develop a secretory profile composed mainly of inflammatory cytokines, chemokines and proteases, a typical signature termed the senescence-associated secretory phenotype (SASP) ${ }^{4}$ or the senescence messaging secretome $(\mathrm{SMS})^{5}$, hereafter referred to as SASP. Some of the SASP factors have cell-autonomous activities that reinforce senescence cell-cycle arrest ${ }^{5}$ and/or promote clearance of senescent cell ${ }^{11,12}$, but other SASP factors have cell non-autonomous functions associated with inflammation and tumorigenesis promotion ${ }^{4}$, indicating that SASP contributes positively and negatively to cancer development, depending on the biological context ${ }^{4,5}$. Because some of the SASP factors, such as IL-6 and PAI- $1^{4,5}$, are known to increase cancer risk in obesity ${ }^{1,13}$, we propose that SASP may contribute to obesity-associated cancer.

To explore this possibility, we first set up a system to examine the impact of dietary obesity on tumorigenesis, using wild-type C57BL/6 mice. However, we were unable to detect a statistically significant difference in cancer development between obese mice fed a high-fat diet (HFD) and lean mice fed a normal diet (data not shown), implying that a certain level of oncogenic stimuli might be required for obesityassociated cancer, especially in wild-type mice maintained in a specific pathogen free (SPF) environment. Because the Ras-pathway is frequently activated in human cancers, including hepatocellular carcinoma $(\mathrm{HCC})^{14}$, we decided to use a treatment with DMBA $(7,12$ dimethylbenz(a)anthracene, a chemical carcinogen that causes an oncogenic Ras mutation) at the neonatal stage, a protocol known to generate a variety of tumours throughout the body ${ }^{15}$. In this setting, we also took advantage of using p21-p-luc mice, in which the expression of the $p 21^{\text {Waf1/Cip } 1}$ gene (a senescence inducer, also known as Cdkn1a) can be monitored noninvasively using a bioluminescence imaging (BLI) technique ${ }^{16}$. The neonatal p21- $p$-luc mice were therefore treated with a single application of DMBA, followed by feeding either HFD or normal diet for 30 weeks (Fig. 1a). Interestingly, a marked increase of the bioluminescent signal was observed in the abdomen of the obese mice, and it originated mainly from liver cancer (Supplementary Fig. 1). Notably, all HFD-fed mice developed HCC, whereas only $5 \%$ of -mice fed normal diet developed malignant tumours in lung, but not liver (Fig. 1b-e and Supplementary Fig. 2). Importantly, moreover, similar HCC development was also observed when genetically obese $\left(o b / o b\right.$, also known as $\left.L e p^{o b / o b}\right)$ mice were treated with DMBA at the neonatal stage (Supplementary Fig. 3a-d), indicating that obesity, but not the HFD, promotes HCC development.

Because the induction of $p 21^{\text {Wafl/Cip } 1}$ expression was observed in liver, particularly in the area of liver cancer (Supplementary Fig. 1c), we speculated that senescent cells might be present in the vicinity of cancerous hepatocytes. Indeed, p2 $1^{\text {Waf1/Cip } 1}$ expression was observed only in activated hepatic stellate cells (HSCs), which express $\alpha$-smooth muscle actin ( $\alpha$-SMA) and desmin $^{7}$ (Fig. 1f). Furthermore, a number of other senescence markers ${ }^{9,10}$, such as p16 ${ }^{\text {INK4a }}$ expression, signs of DNA damage (53BP1 foci and $\gamma \mathrm{H} 2 \mathrm{AX}$ foci) and inhibited cell proliferation (the absence of bromodeoxyuridine incorporation and Ki-67 expression), were also observed in activated HSCs despite absence of oncogenic ras mutation (Fig. If and Supplementary Figs $3 \mathrm{e}$ and 4). Interestingly, moreover, increased expression of IL-6, Gro- $\alpha$, CXCL9 (major components of SASP) ${ }^{4,5}$, but not HGF (a differentiation marker) ${ }^{7}$, was observed in activated HSCs, but not in other types of liver cells (Fig. 1f and Supplementary Figs $3 e$ and 5), indicating that these activated HSCs are senescing and may promote obesity-associated HCC development via SASP. It should be noted that, unlike the study using carbon tetrachloride $\left(\mathrm{CCl}_{4}\right)^{11}$, fibrosis was not apparent in HFD-fed mice (Supplementary Fig. 6), precluding the possibility that the appearance of senescent HSCs was a by-product of liver fibrosis.

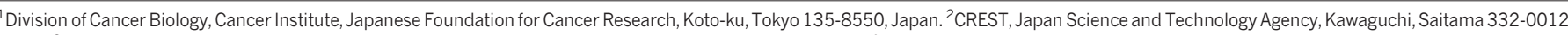

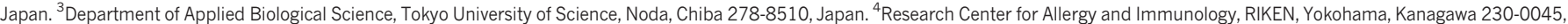

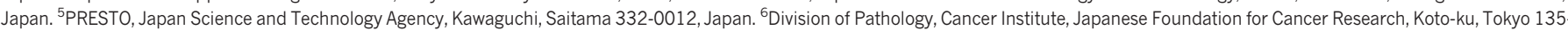

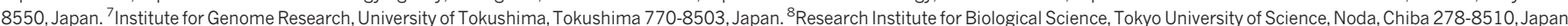
${ }^{9}$ Graduate School of Frontier Sciences, University of Tokyo, Kashiwa, Chiba 277-8561, Japan. ${ }^{10}$ School of Veterinary Medicine, Azabu University, Sagamihara, Kanagawa $229-8501$, Japan.

*These authors contributed equally to this work 


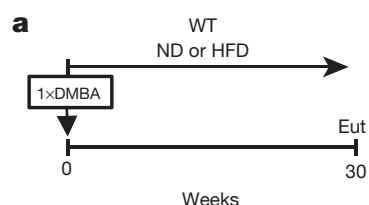

b
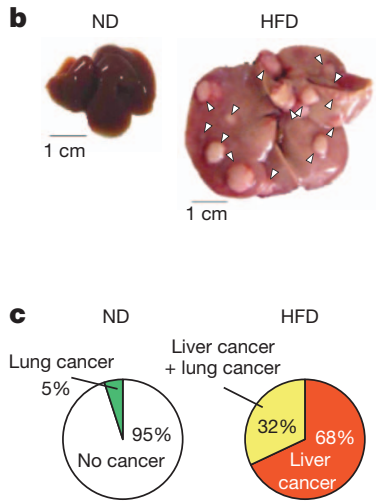

d

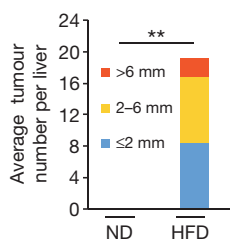

e

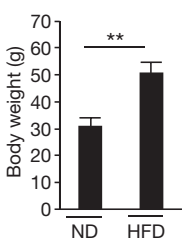

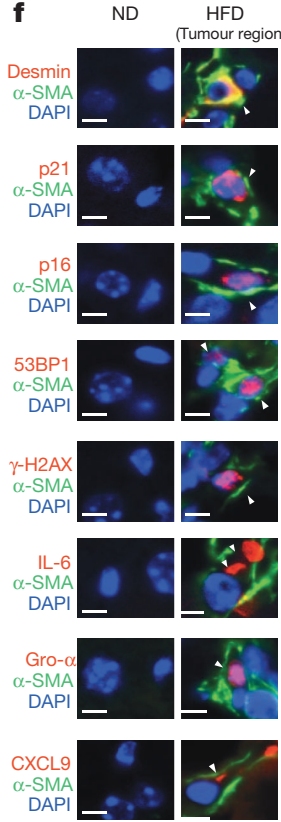

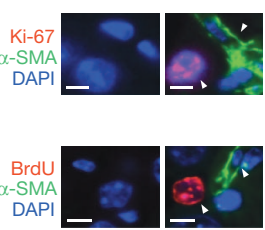

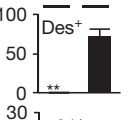

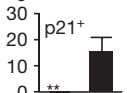

30

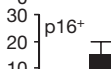

$10-1$

30 53BP1+

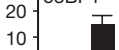

0

$30] \gamma-\mathrm{H}_{2} \mathrm{AX}^{+}$

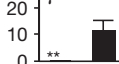

0

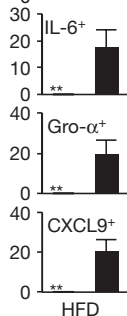

HFD

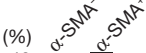

$40 \frac{\sqrt{\mathrm{Ki}-67+}}{\mathrm{d}}$

40 Ki-67+

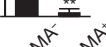

$30 \stackrel{d^{5}}{0^{5}}$

$20 \mathrm{BrdU}^{+}$

10 工茫

Figure 1 Cellular senescence in HSCs. a, Timeline of the experimental procedure ( $n=19$ per group). Eut, euthanasia; ND, normal diet.

b, Representative macroscopic photographs of livers. Arrowheads indicate HCCs. c, The ratios of cancer formation. $\mathbf{d}$, The average liver tumour numbers and their relative size distribution. e, The average body weights at the age of 30 weeks. f, Immunofluorescence analysis of liver section. HSCs were visualized by $\alpha$-SMA staining and DNA was stained by $4^{\prime}, 6$-diamidino-2-phenylindole (DAPI). Scale bars, $2.5 \mu \mathrm{m}$. Arrowheads indicate $\alpha$-SMA expressing cells that were positive for indicated markers. The histograms indicate the percentages of $\alpha$-SMA-expressing cells that were positive for indicated markers. At least 100 cells were scored per group. For all graphs, error bars indicate mean \pm standard deviation (s.d.). $* * P<0.01$.

To ascertain the role of SASP in obesity-associated HCC development, we next sought evidence that the blockage of SASP can reduce obesity-associated HCC development. Although we were unable to detect the expression of IL- $1 \alpha$ (an upstream regulator of SASP induction $)^{8}$ in HSCs, significant induction of IL-1 $\beta$ (a functional homologue of IL-1 $\alpha$ ) and its activator, caspase-1 (an essential component of the inflammasome), was observed in senescent HSCs (Fig. 2a-c). Moreover, the addition of recombinant IL- $1 \beta$ caused the dose-dependent induction of Il-6 and Gro- $\alpha$ (also known as Cxcl1) gene expression in cultured primary murine HSCs (Supplementary Fig. 7a), indicating that inflammasome activation and subsequent IL-1 $\beta$ maturation can act as an upstream regulator of SASP induction in HSCs. Indeed, the levels of SASP factor expression in activated HSCs were substantially diminished in mice lacking the $I l-1 \beta$ gene $\left(I l-1 \beta^{-1-}\right.$ mice, also known as $I l 1 b^{-1-}$ ) (Fig. 2c), and the numbers and sizes of the liver tumours that developed in $I l-1 \beta^{-1-}$ mice were strikingly reduced, as compared with wild-type mice (Fig. $2 \mathrm{~d}, \mathrm{e}$ ), although the degree of steatohepatitis was not attenuated (Supplementary Fig. 8a, b). It should be noted, however, that other senescence markers, such as 53BP1 foci, $\mathrm{p} 21^{\text {Waf1/Cip } 1}$ expression and inhibited cell proliferation, were still observed in the activated HSCs of $\mathrm{Il}-1 \beta^{-1-}$ mice (Fig. 2c and Supplementary Fig. 8c). These results are somewhat consistent with a recent observation that the

a
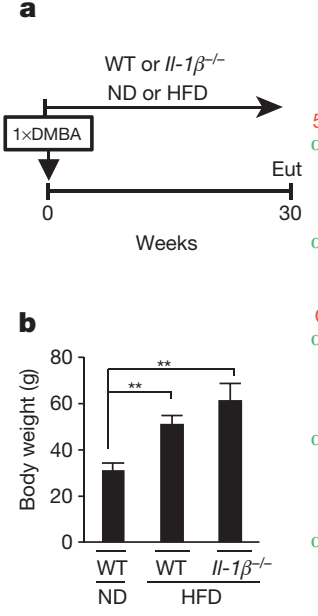

c
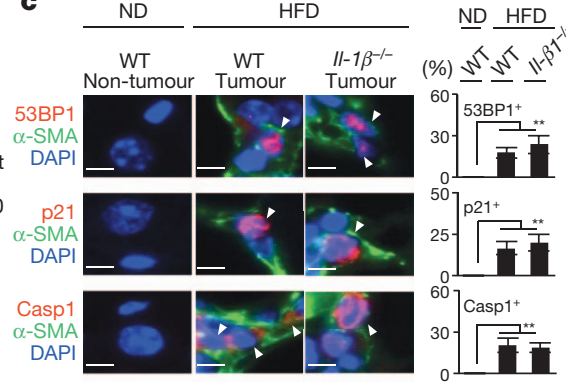

${ }^{60}{ }^{6}{ }^{2} 1^{+}$
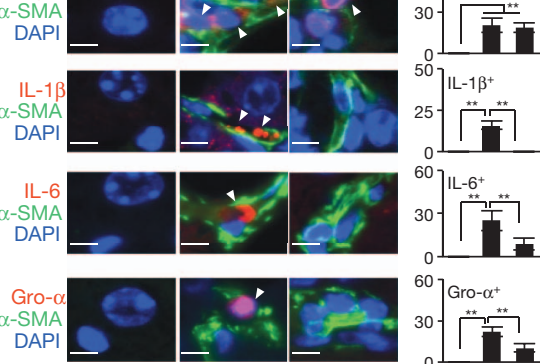

$\left.{ }^{50}\right] \mathrm{IL}-1 \beta^{+}$

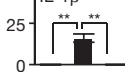

607 IL-6 $^{+}$
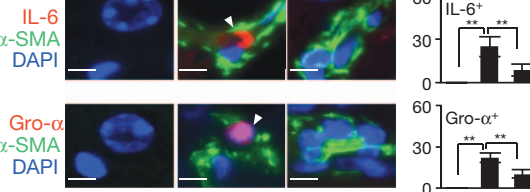

${ }^{60}$ Gro- $\alpha^{+}$

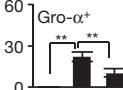

d
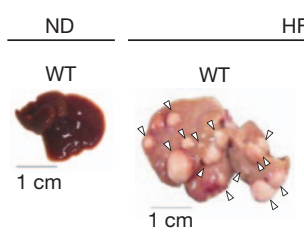

HFD
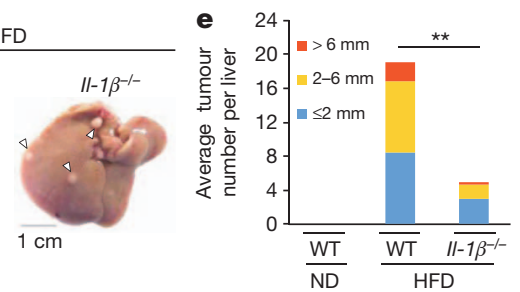

Figure $2 \mid$ IL-1 $\beta$ deficiency alleviates obesity-induced HCC development. a, Timeline of the experimental procedure (wild type (WT), $n=19$; $\left.I l-1 \beta^{-l-}, n=9\right)$. $\mathbf{b}$, The average body weights at the age of 30 weeks. c, Immunofluorescence analysis of liver sections. HSCs were visualized by $\alpha$-SMA staining and DNA was stained by DAPI. Scale bars, $2.5 \mu \mathrm{m}$. The histograms indicate the percentages of $\alpha$-SMA-expressing cells that were positive for indicated markers. At least 100 cells were scored per group. d, Representative macroscopic photographs of livers. Arrowheads indicate HCCs. e, The average liver tumour numbers and their relative size distribution. For all graphs, error bars indicate mean \pm s.d. $* * P<0.01$.

expression of $\mathrm{p} 21^{\mathrm{Waf} 1 / \mathrm{Cip} 1}$ can induce senescence cell-cycle arrest without SASP induction ${ }^{17}$, suggesting that SASP, but not senescence cellcycle arrest, promotes obesity-associated HCC development.

To further verify this idea, we next attempted to deplete senescent HSCs from obese wild-type mice treated with DMBA at the neonatal stage. As reported previously ${ }^{18}$, an intravenous injection of liposomes carrying small interfering RNA (siRNA) against HSP47 substantially reduced the abundance of activated HSCs, coinciding with a significant reduction of HCC development (Supplementary Fig. 9a-f). Note that this was not accompanied by an attenuation of steatohepatitis (Supplementary Fig. 9g, h). These results, along with the data from Il-1 $\beta^{-/-}$mice (Fig. 2), strongly indicate that senescent HSCs have enhancing roles in HCC development via SASP, at least in the neonatal DMBA plus obesity-induced HCC model. It is also noteworthy that neither the deletion of the $I l-1 \beta$ gene nor the depletion of senescent HSCs caused appreciable weight loss (Fig. $2 \mathrm{~b}$ and Supplementary Fig. 9c), implying that there may be an indirect link between obesity and HCC development, at least in this experimental setting. These observations then raised the question of how obesity provokes SASP in HSCs.

Emerging evidence has indicated that alterations of intestinal microbiota are associated with obesity ${ }^{19}$. Furthermore, the activation of toll-like receptor (TLR) 4 by lipopolysaccharide (LPS) from intestinal Gram-negative bacteria has been shown to promote HCC development, in an HCC model using DEN (diethyl nitrosamine) plus $\mathrm{CCl}_{4}$ treatment ${ }^{20}$. We thus explored the possibility that intestinal bacteria have key roles in obesity-associated HCC development. Indeed, a 
treatment with a well-established oral antibiotic cocktail (4Abx), which reduces the number of commensal intestinal bacteria ${ }^{20}$, caused a marked reduction of HCC development, accompanied by a marked decrease in senescent HSCs in the neonatal DMBA plus obesityinduced HCC model (Fig. 3 and Supplementary Fig. 3). As reported ${ }^{20}$, $4 \mathrm{Abx}$ treatment resulted in not only a $>99.5 \%$ reduction of the presence of bacterial $16 \mathrm{~S}$ ribosomal RNA gene in faeces, but also an enlargement of caecum commonly observed in germ-free mice (Fig. 3b and data not shown). Unexpectedly, however, a slight increase, rather than decrease, in HCC development was observed in mice lacking the Tlr4 gene (Tlr4 ${ }^{-/-}$) (Supplementary Fig. 10), indicating that LPS from Gram-negative bacteria is unlikely to promote HCC development in this setting. Indeed, meta $16 \mathrm{~S}$ rRNA gene sequencing analysis of the intestinal microbiota revealed that the percentage of Gram-positive bacterial strains indigenous to the human and rodent intestinal tracts $^{6}$ was dramatically increased with a HFD (Fig. 4a). Moreover, a treatment with vancomycin (VCM), an antibiotic that preferentially targets Gram-positive bacteria, alone was sufficient to block HCC development and the appearance of senescent HSCs (Figs 3d-f, 4a and Supplementary Fig. 3). These results lead us to propose that the obesity-associated increase of Gram-positive bacteria may promote HCC development, presumably through the enterohepatic circulation of gut bacterial metabolites or toxins.

To substantiate this idea, the serum metabolites of HFD- and normaldiet-fed mice were analysed by liquid chromatography mass spectrometry (LC-MS). Interestingly, the level of deoxycholic acid (DCA), a secondary bile acid produced solely by the $7 \alpha$-dehydroxylation of a

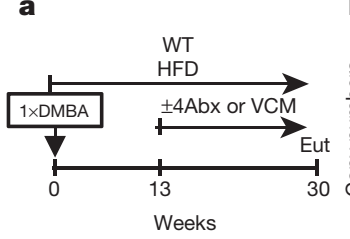

b
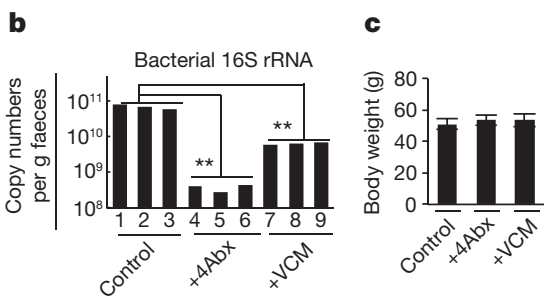
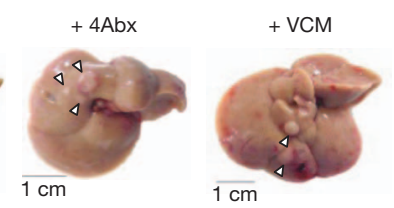

$1 \mathrm{~cm}$
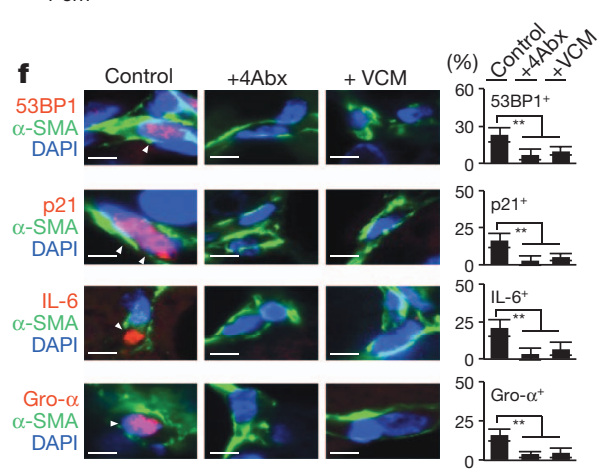

e

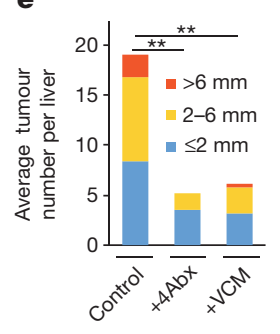

d

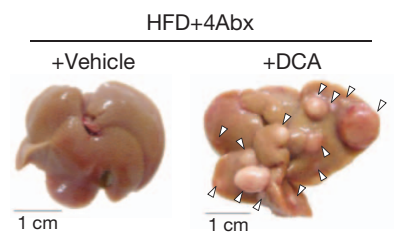
mean \pm s.d. $* P<0.05, * * P<0.01$.
$+4 A b x$ e

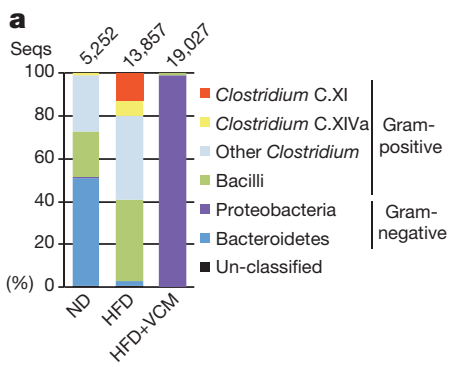

b

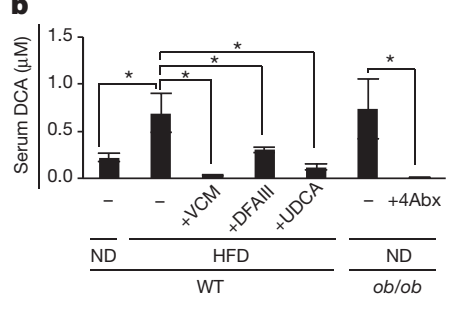

c

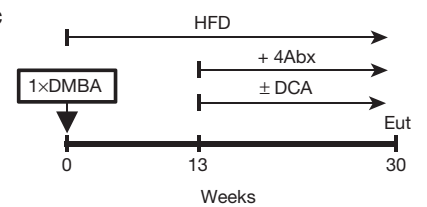

9
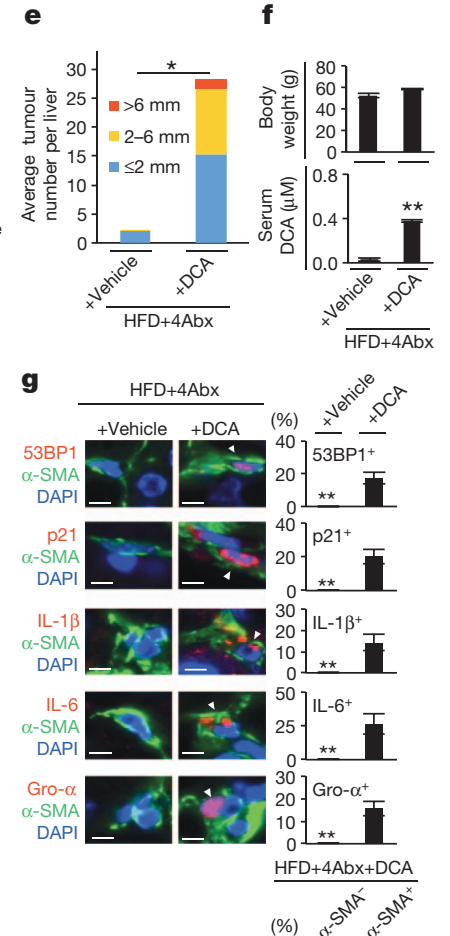
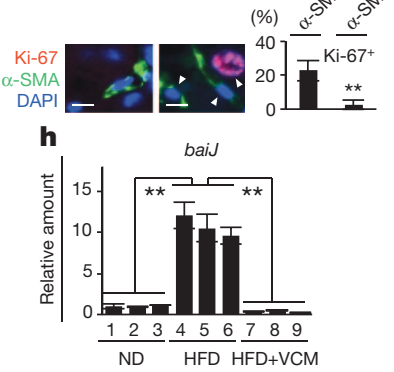

Figure 4 Bacterial metabolite promotes obesity-induced HCC

development. a, The relative abundance of OTUs (\%) in the faecal bacterial community. Data are representative of five mice per group. $\mathbf{b}$, Serum DCA concentration (ND, $n=4$; HFD, $n=6$; HFD+VCM, $n=3$; HFD+DFAIII, $n=3$; HFD+UDCA, $n=3 ; o b / o b, n=3 ; o b / o b+4 \mathrm{Abx}, n=3)$. Error bars indicate mean \pm s.e.m. c, Timeline of the experimental procedure $(n=3$ per group). d, Representative macroscopic photographs of livers. Arrowheads indicate HCCs. e, The average tumour numbers and their relative size distribution. f, The average body weight and serum DCA concentration. g, Immunofluorescence analysis of liver sections. Scale bars, $2.5 \mu \mathrm{m}$. The histograms indicate the percentages of $\alpha$-SMA-expressing cells that were positive for indicated markers. At least 100 cells were scored per group. h, The quantitative real time PCR (qPCR) analysis of baiJ gene in the faeces (180 mg) of indicated mice used in $\mathbf{a}$. For all graphs except $\mathbf{b}$, error bars indicate

primary bile acids carried out by gut bacteria such as strains belonging to Clostridium cluster XI and XIVa ${ }^{6}$ (VCM-sensitive Gram-positive bacteria), was substantially increased by the HFD feeding, and was reduced by antibiotic treatments (Figs $3 \mathrm{a}$ and $4 \mathrm{~b}$ ). Note that DCA is known to cause DNA damage through reactive oxygen species production ${ }^{21}$ and DNA damage is a critical inducer of SASP ${ }^{4,22}$. Moreover, in addition to colon carcinogenesis ${ }^{23}$, DCA has been shown to enhance liver carcinogenesis $^{24}$. These notions prompted us to examine if DCA has key roles in obesity-associated HCC development. To this end, we attempted to lower the levels of DCA, by either decreasing the $7 \alpha$ dehydroxylation activity with difructose anhydride III (DFA III $)^{25}$ or stimulating bile acid secretion with ursodeoxycholic acid (UDCA) ${ }^{26}$. Notably, lowering the DCA concentration substantially reduced HCC development, accompanied by a marked decrease in senescent HSCs in obese mice treated with DMBA at the neonatal stage (Fig. $4 \mathrm{~b}$ and
Figure $3 \mid$ Antibiotics treatments alleviate obesity-induced HCC development. a, Timeline of the experimental procedure (HFD, $n=19$; HFD $+4 \mathrm{Abx}, n=12$; HFD + VCM, $n=6$ ). $\mathbf{b}$, The copy number of intestinal bacteria in faeces of indicated mice. $c$, The average body weights at the age of 30 weeks. d, Representative macroscopic photograph of livers. Arrowheads indicate HCCs. e, The average tumour numbers and their relative size distribution. f, Immunofluorescence analysis of liver sections. HSCs were visualized by $\alpha$-SMA staining and DNA was stained by DAPI. Scale bars, $2.5 \mu \mathrm{m}$. The histograms indicate the percentages of $\alpha$-SMA expressing cells that were positive for indicated markers. At least 200 cells were scored per group. For all graphs, error bars indicate mean \pm s.d. $* * P<0.01$. 
Supplementary Figs 11 and 12). In a reciprocal set of experiments, we also assessed whether DCA-feeding enhances HCC development in mice treated with DMBA at the neonatal stage (Fig. 4c). Intriguingly, although DCA feeding alone was insufficient to enhance HCC development in lean mice fed a normal diet at 30 weeks (data not shown), a significant enhancement of HCC development (Fig. 4c-f), accompanied by the appearance of senescence cell-cycle arrest and SASP in HSCs (Fig. 4g), was observed when HFD-fed mice treated with 4 Abx were fed DCA for 17 weeks.

Notably, operational taxonomic unit (OTU)-based bacterial diversity analysis (Fig. 4a), in conjunction with a quantitative PCR analysis (Supplementary Fig. 13), revealed that the population of cluster XI of the genus Clostridium was strikingly increased in HFD-fed mice. Interestingly, phylogenetic analysis of the bacterial OTUs revealed that the population of Clostridium cluster XI is composed of a single bacterial taxon (OTU-1105) close to the DCA-producing strain Clostridium sordellii, and represents more than $12 \%$ of the faecal bacteria in HFD-fed mice (Supplementary Fig. 14). Concordantly, moreover, the abundance of the baiJ gene, a gene involved in bile acid $7 \alpha$-dehydroxylation ${ }^{27}$, was remarkably increased in faeces of mice fed HFD and was reduced by VCM treatment (Fig. 4h). On the other hand, a bacterial taxon (OTU-154) close to other DCA producing strains belonging to Clostridium cluster XIVa (Clostridium hylemonae and Clostridium scindens) represents only $0.5 \%$ of the total faecal bacteria in HFD-fed mice (Supplementary Fig. 14). Thus, although other bacteria may also be involved here, the simplest explanation for our data is that OTU-1105 belonging to Clostridium cluster XI contribute to an increase in the DCA level at least to some extent in HFD-fed mice.

Finally, to further support and extend our murine data to human biology, we tested whether IL- $1 \beta$ treatment can induce SASP in cultured primary human HSCs. As in murine HSCs, the addition of recombinant IL- $1 \beta$ caused the induction of $I l-6$ and $I l-8$ (a functional homologue of murine $G r o-\alpha$ ) gene expression in cultured primary human HSCs (Supplementary Fig. 7b). Importantly, moreover, signs of cellular senescence and SASP were also observed in the HSCs without serious fibrosis in the area of HCC arising in patients with nonalcoholic steatohepatitis (NASH) ${ }^{3}$ (8 out of 26) (Supplementary Fig. 15). This is somewhat consistent with previous observations that replicative senescence of cultured human HSCs is accompanied by a pronounced inflammatory but less fibrogenic phenotype ${ }^{28}$ and a certain percentage of NASH- associated HCC arose from the non-cirrhotic liver ${ }^{29}$. Unlike rodents, the human liver cannot $7 \alpha$-hydroxylate DCA, forming cholic acid ${ }^{6}$. Hence, DCA can accumulate to very high levels $(>50 \%)$ in the bile acid pool of humans ${ }^{6}$. These data, together with the previous observation that high fat consumption resulted in higher faecal DCA concentrations in healthy male volunteers (ages 20-60) ${ }^{30}$, suggest that DCA-induced senescent HSCs may contribute to at least certain aspects of obesity-associated HCC development via SASP in humans as well.

It should be noted that although many of the perturbations, for example, the $I l-1 \beta$ knockout, antibiotics treatment and lower DCA levels, significantly prevent HCC development, residual HCCs were still observed with these perturbations (Figs $2 \mathrm{e}$ and $3 \mathrm{e}$ and Supplementary Figs $11 \mathrm{c}$ and $12 \mathrm{c}$ ). These results, in conjunction with the observation that DCA-feeding alone was insufficient to enhance HCC development in lean mice fed a normal diet until at least 30 weeks (data not shown), imply that an additional factor associated with obesity may exist to promote obesity-associated HCC development. Nevertheless, combining published data ${ }^{14,19,21,24,30}$ with our findings, it is clear that the increased levels of DCA produced by gut bacteria play key roles in the promotion of obesity-associated HCC development via provoking SASP in HSCs, at least in the neonatal DMBA plus obesityinduced HCC model (Supplementary Fig. 16). A greater understanding of the molecular mechanisms linking gut microbial metabolite to SASP will therefore provide valuable new insights into how to bypass this undesirable side effect of cellular senescence.

\section{METHODS SUMMARY}

Chemically-induced carcinogenesis. DMBA treatments ${ }^{15}$ consisted of a single application of $50 \mu \mathrm{l}$ of a solution $0.5 \%$ DMBA $(7,12$-dimethylbenz [a] anthracene, Sigma) in acetone to the dorsal surface on postnatal day $4-5$. Mother mice with pups were then fed either normal diet or HFD until weaning. At the age of 4 weeks old, pups were weaned and continuously fed either normal diet or HFD until euthanized.

Bacterial 16S rRNA amplicon sequencing and analysis. Bacterial genomic DNA was isolated from mice faeces, amplified for V1-V4 hypervariable regions of the $16 \mathrm{~S}$ rRNA gene, and used for pyrosequencing analysis.

Full Methods and any associated references are available in the online version of the paper.

Received 10 December 2012; accepted 4 June 2013.

Published online 26 June 2013.

1. Khandekar, M. J., Cohen, P. \& Spiegelman, B. M. Molecular mechanisms of cancer development in obesity. Nature Rev. Cancer 11, 886-895 (2011).

2. Calle, E. E. \& Kaaks, R. Overweight, obesity and cancer: epidemiological evidence and proposed mechanisms. Nature Rev. Cancer 4, 579-591 (2004)

3. Sun, B. \& Karin, M. Obesity, inflammation, and liver cancer. J. Hepatol. 56, 704-713 (2012).

4. Coppé, J. P. et al. Senescence-associated secretory phenotypes reveal cellnonautonomous functions of oncogenic RAS and the p53 tumor suppressor. PLoS Biol. 6, 2853-2868 (2008).

5. Kuilman, T. \& Peeper, D. S. Senescence-messaging secretome: SMS-ing cellular stress. Nature Rev. Cancer 9, 81-94 (2009).

6. Ridlon, J. M., Kang, D. J. \& Hylemon, P. B. Bile salt biotransformations by human intestinal bacteria. J. Lipid Res. 47, 241-259 (2006).

7. Friedman, S. L. Hepatic stellate cells: protean, multifunctional, and enigmatic cells of the liver. Physiol. Rev. 88, 125-172 (2008).

8. Orjalo, A. V., Bhaumik, D., Gengler, B. K., Scott, G. K. \& Campisi, J. Cell surface-bound IL- $1 \alpha$ is an upstream regulator of the senescence-associated IL-6/IL-8 cytokine network. Proc. Natl Acad. Sci. USA 106, 17031-17036 (2009).

9. Collado, M. \& Serrano, M. Senescence in tumours: evidence from mice and humans. Nature Rev. Cancer 10, 51-57 (2010).

10. Kuilman, T., Michaloglou, C., Mooi, W. J. \& Peeper, D. S. The essence of senescence. Genes Dev. 24, 2463-2479 (2010).

11. Krizhanovsky, V. etal. Senescence of activated stellate cells limits liver fibrosis. Cell 134, 657-667 (2008)

12. Kang, T.W. etal. Senescence surveillance of pre-malignant hepatocytes limits liver cancer development. Nature 479, 547-551 (2011).

13. Park, E. J. et al. Dietary and genetic obesity promote liver inflammation and tumorigenesis by enhancing IL-6 and TNF expression. Cell 140, 197-208 (2010).

14. Newell, P. et al. Ras pathway activation in hepatocellular carcinoma and antitumoral effect of combined sorafenib and rapamycin in vivo. J. Hepatol. 51, 725-733 (2009).

15. Serrano, M. et al. Role of the INK4a locus in tumor suppression and cell mortality Cell 85, 27-37 (1996).

16. Ohtani, N. et al. Visualizing the dynamics of $\mathrm{p} 21^{\text {Waf1/Cip } 1} \mathrm{cyclin}$-dependent kinase inhibitor expression in living animals. Proc. Natl Acad. Sci. USA 104, 15034-15039 (2007).

17. Coppé, J.-P. et al. Tumor suppressor and aging biomarker $\mathrm{p} 16^{\mathrm{INK} 4 \mathrm{a}}$ induces cellular senescence without the associated inflammatory secretory phenotype. J. Biol. Chem. 286, 36396-36403 (2011).

18. Sato, Y. et al. Resolution of liver cirrhosis using vitamin A-coupled liposomes to deliver siRNA against a collagen-specific chaperone. Nature Biotechnol. 26, 431-442 (2008).

19. Ley, R. E., Turnbaugh, P. J., Klein, S. \& Gordon, J. I. Microbial ecology: human gut microbes associated with obesity. Nature 444, 1022-1023 (2006).

20. Dapito, D. H. et al. Promotion of hepatocellular carcinoma by the intestinal microbiota and TLR4. Cancer Cell 21, 504-516 (2012).

21. Payne, C. M. et al. Deoxycholate induces mitochondrial oxidative stress and activates NF-kB through multiple mechanisms in HCT-116 colon epithelial cells. Carcinogenesis 28, 215-222 (2007).

22. Takahashi, A. et al. DNA damage signaling triggers degradation of histone methyltransferases through APC/C ${ }^{C d h 1}$ in senescent cells. Mol. Cell 45, 123-131 (2012).

23. McGarr, S. E., Ridlon, J. M. \& Hylemon, P. B. Diet, anaerobic bacterial metabolism, and colon cancer: a review of the literature. J. Clin. Gastroenterol. 39, 98-109 (2005).

24. Kitazawa, S. et al. Enhanced preneoplastic liver lesion development under 'selection pressure' conditions after administration of deoxycholic or lithocholic acid in the initiation phase in rats. Carcinogenesis 11, 1323-1328 (1990).

25. Minamida, K., Ohashi, M., Hara, H., Asano, K. \& Tomita, F. Effects of ingestion of difructose anhydride III (DFA III) and the DFA III-assimilating bacterium Ruminococcus productus on rat intestine. Biosci. Biotechnol. Biochem. 70, 332-339 (2006).

26. Beuers, U. Drug insight: mechanisms and sites of action of ursodeoxycholic acid in cholestasis. Nat. Clin. Pract. Gastroenterol. Hepatol. 3, 318-328 (2006).

27. Ridlon, J. M. \& Hylemon, P. B. Identification and characterization of two bile acid coenzyme A transferases from Clostridium scindens, a bile acid $7 \alpha$-dehydroxylating intestinal bacterium. J. Lipid Res. 53, 66-76 (2012). 
28. Schnabl, B., Purbeck, G. A., Choi, Y. H., Hagedorn, C. H. \& Brenner, D. A. Replicative senescence of activated human hepatic stellate cells is accompanied by a pronounced inflammatory but less fibrogenic phenotype. Hepatology 37, 653-664 (2003).

29. Takuma, Y. \& Nouso, K. Nonalcoholic steatohepatitis-associated hepatocellular carcinoma: our case series and literature review. World J. Gastroenterol. 16, 1436-1441 (2010).

30. Rafter, J. J. et al. Cellular toxicity of fecal water depends on diet. Am. J. Clin. Nutr. 45, 559-563 (1987).

Supplementary Information is available in the online version of the paper.

Acknowledgements The authors thank M. Oshima for suggestions in antibiotics treatment and members of the Hara laboratory for discussion during the preparation of this manuscript. This work was supported by grants from Japan Science and Technology Agency (JST), Ministry of Education, Culture, Sports, Science and Technology of Japan (MEXT), Ministry of Health, Labour and Welfare of Japan (MHLW) Uehara Memorial Foundation and Takeda Science Foundation. S.Y. was partly supported by a postdoctoral fellowship from the Japan Society for Promotion of Science (JSPS). T.M.L. was partly supported by an international scholarship from the Ajinomoto Scholarship Foundation.

Author Contributions E.H. and N.O. designed the experiments, analysed the data and wrote the manuscript. N.O., S.Y. and T.M.L. performed obesity-induced liver cancer experiments. K.A., K.O., H.M., M.H. and K.H. performed bacterial genome data analysis. H.K., S.S. and Y.I. performed histopathological analysis of mouse and human liver cancer specimens. S.O. performed metabolite analysis. Y.I. provided $/ /-1 \beta^{-/-}$mice. E.H oversaw the projects.

Author Information Bacterial 16S rRNA amplicon sequence data have been deposited in DDBJ (http://www.ddbj.nig.ac.jp/index-e.html) with the accession number DRA000952. Reprints and permissions information is available at www.nature.com/ reprints. The authors declare no competing financial interests. Readers are welcome to comment on the online version of the paper. Correspondence and requests for materials should be addressed to E.H. (eiji.hara@jfcr.or.jp). 


\section{METHODS}

Mice and diet. The $p 21-p-l u c$ mice $(\mathrm{CD} 1)^{16}$ were backcrossed with C57BL/6 mice for eight generations. The leptin-deficient $(o b / o b)$ mice (C57BL/6) were purchased from Charles River Laboratories Japan, Inc. $\mathrm{Tlr}^{-/-}$mice (C57BL/6) were purchased from Oriental Bioservices. Il-1 $\beta^{-/-}$mice (C57BL/6) were provided by $\mathrm{Y}$. Iwakura $^{31}$. Male mice were used for all the experiments in this study. The mice were maintained under specific pathogen-free (SPF) conditions, on a 12-h lightdark cycle, and fed normal diet (CE-2 from CLEA Japan Inc., composed of $12 \mathrm{kcal} \%$ fat, $29 \mathrm{kcal} \%$ protein, $59 \mathrm{kcal} \%$ carbohydrates) or high-fat diet (HFD, D12492 from Research Diets Inc., composed of $60 \mathrm{kcal} \%$ fat, $20 \mathrm{kcal} \%$ protein, $20 \mathrm{kcal} \%$ carbohydrates) ad libitum. Mice with more than $45 \mathrm{~g}$ weight at the age of 30 weeks old were used as obese mice for all the experiments. We measured the amount of food our mice eat and found that a $50 \mathrm{~g}$ HFD mouse eats $3.44 \mathrm{~g}$ food a day. This equates to $1.2 \mathrm{~g}$ of fat per day or $24 \mathrm{~g}$ per $\mathrm{kg}$. According to the ReaganShaw equation ${ }^{32}$ (human equivalent dose $\left(\mathrm{mg} \mathrm{kg}^{-1}\right)=$ mouse dose $\left(\mathrm{mg} \mathrm{kg}^{-1}\right) \times$ mouse $K_{\mathrm{m}}$ factor $\div$ human $K_{\mathrm{m}}$ factor; where the mouse and human $K_{\mathrm{m}}$ factors are 3 and 37 , respectively), this is equivalent to a $70 \mathrm{~kg}$ human eating $136 \mathrm{~g}$ of fat a day. The sample size used in this study was determined based on the expense of data collection, and the need to have sufficient statistical power. Randomization and blinding were not used in this study. All animal experiments were cared for by protocols approved by the Committee for the Use and Care of Experimental Animals of the Japanese Foundation for Cancer Research (JFCR).

Chemically induced carcinogenesis. DMBA treatments ${ }^{15}$ consisted of a single application of $50 \mu \mathrm{l}$ of a solution $0.5 \%$ DMBA (7,12-dimethylbenz(a)anthracene, Sigma) in acetone to the dorsal surface on postnatal day 4-5. After this application, mother mice with pups were fed normal diet or HFD. At the age of 4 weeks old, pups were weaned and continuously fed either normal diet or HFD until euthanized. Evaluation of tumour number and size was determined by counting the number of visible tumours and measuring the size of the tumour.

Bioluminescence imaging. Bioluminescence imaging was performed as previously described ${ }^{16,33}$. In brief, for the detection of luciferase expression, mice were anesthetized, injected intraperitoneally with D-luciferin sodium salt $\left(75 \mathrm{mg} \mathrm{kg}^{-1}\right)$ $5 \mathrm{~min}$ before beginning photon recording. Mice were placed in the light-tight chamber and a grey-scale image of the mice was first recorded with dimmed light followed by acquisition of luminescence image using a cooled charged-coupled device (CCD) camera (PIXIS 1024B; Princeton Instruments). The signal-to-noise ratio was increased by $2 \times 2$ binning and $5 \mathrm{~min}$ exposure. For colocalization of the luminescent photon emission on the animal body, grey scale and pseudo-colour images were merged by using IMAGE-PRO PLUS (Media Cybernetics).

Antibiotics treatment. Antibiotics treatment was performed as previously described $^{20}$ using a combination of four antibiotics (4Abx) of ampicillin $\left(1 \mathrm{gl}^{-1}\right)$, neomycin $\left(1 \mathrm{gl}^{-1}\right)$, metronidazole $\left(1 \mathrm{gl}^{-1}\right)$ and vancomycin $\left(500 \mathrm{mgl}^{-1}\right)$, or vancomycin $\left(500 \mathrm{mgl}^{-1}\right)$ alone $(\mathrm{VCM})$ in drinking water at the age of 13 weeks old until killed.

Histology and immunofluorescence analysis. Haematoxylin and eosin staining and immunofluorescence analysis were performed as previously described ${ }^{16}$. The primary antibodies used for mouse samples were as follows: $\alpha$-SMA (Sigma A5228), desmin (abcam ab15200), p21 (abcam ab2961), p16 (Santa Cruz sc1207), 53BP1 (Santa Cruz sc22760), $\gamma$-H2AX (CST 9718), IL-6 (abcam ab6672), Gro- $\alpha$ (abcam ab17882), Ki-67 (Thermo RM9106), bromodeoxyuridine (abcam ab6326), caspase-1 (Millipore 06-503), IL-1 $\beta$ (R\&D systems AF-401-NA), HSP47 (Santa Cruz sc8352), CXCL9 (abcam ab137792), F4/80 (Invitrogen BM8) and CD45(Millipore 05-1416). The primary antibodies used for human samples were as follows: $\alpha$-SMA (Dako M0851), $\gamma$-H2AX (CST 9718), p16 (Santa Cruz sc56330), p21 (CST \#2947), IL-6 (abcam ab6672), IL-8 (abcam ab18672), 53BP1 (Santa Cruz sc22760) and caspase-1 (Millipore 06-503).

Quantitative PCR. Total RNA was extracted from mouse tissues using TRIzol reagent (Life technologies) and reverse transcription and quantitative PCR were performed as previously described ${ }^{22}$. Primers used were as follows: human GAPDH, 5'-CAACTACATGGTTTACATGTTC-3' (forward) and 5'-GCCAGTGGACT CCACGAC-3' (reverse), mouse Gapdh, 5'-CAACTACATGGTCTACATGTTC$3^{\prime}$ (forward) and 5' -CACCAGTAGACTCCACGAC-3' (reverse), human $I L-6,5^{\prime}$ CTCGACGGCATCTCAGCCCTGA-3' (forward) and 5'-CTGCCAGTGCCTC TTTGCTGCTTT-3' (reverse), mouse $\mathbf{I l - 6 , 5}$ ' -TGATTGTATGAACAACGATG ATGC-3' (forward) and 5'-GGACTCTGGCTTTGTCTTTCTTGT-3' (reverse), human $I L-8,5^{\prime}$-AAGGAAAACTGGGTGCAGAG-3' (forward) and $5^{\prime}$-ATTGC ATCTGGCAACCCTAC- $3^{\prime}$ (reverse), mouse Gro- $\alpha, 5^{\prime}$-GCTGGGATTCACC TCAAGAA-3' (forward) and 5'-AGGTGCCATCAGAGCAGTCT-3' (reverse), bacterial baij $5^{\prime}$-TCAGGACGTGGAGGCGATCCA-3' (forward) and $5^{\prime}$ TACRTGATACTGGTAGCTCCA-3' (reverse), Clostridium cluster XI $16 \mathrm{~S}$ rRNA gene 5'-TGACGGTACYYNRKGAGGAAGCC-3' (forward) and 5'-ACT ACGGTTRAGCCGTAGCCTTT-3' (reverse).
In vivo RNAi experiment. $250 \mu \mathrm{l}$ of siRNA solution $\left(3 \mathrm{mg} \mathrm{ml}^{-1}\right)$ against HSP47 or control siRNA were mixed with $250 \mu \mathrm{l}$ of complexation buffer and $500 \mu \mathrm{l}$ of Invivofectamine (Life Technologies), incubated for $30 \mathrm{~min}$ at $50^{\circ} \mathrm{C}$, and dialysed at room temperature for $2 \mathrm{~h}$ in 11 of PBS (pH7.4). Dialysed siRNAInvivofectamine complex was collected and $3 \mu \mathrm{g}$ per g (weight) was injected through mice's tail vein twice a week for 15 weeks until killed. The sequences of HSP47 targeting oligo are as follows. 5'-GCACUGCUUGUGAACGCCAU GUUCU-3' (sense), 5'-AGAACAUGGCGUUCACAAGCAGUGC-3' (antisense). As a negative control, Ambion In vivo Negative Control \#1 siRNA(4457289) was used.

Treatment with DCA, UDCA and DFAIII. Deoxycholic acid (DCA) was dissolved in absolute ethanol and diluted in $66 \%$ propylene glycol to reduce the concentration of alcohol to 5\%. HFD-fed mice treated with DMBA at neonatal stage were fed a combination of four antibiotics (4Abx) with $40 \mu \mathrm{g}$ per $\mathrm{g}$ (weight) of DCA or vehicle (control) three times per week using a plastic feeding tube at the age of 13 weeks old until killed. Ursodeoxycholic acid (UDCA) tablets (TanabeMitsubishi Pharma) were powdered and dissolved in $66 \%$ propylene glycol. HFDfed mice treated with DMBA at neonatal stage were fed $60 \mu \mathrm{g}$ per $\mathrm{g}$ (weight) of UDCA or vehicle (control) using a plastic feeding tube every day at the age of 15 weeks old until killed. Difructose anhydride III (DFAIII)was dissolved in saline. HFD-fed mice treated with DMBA at neonatal stage were fed $0.1 \mathrm{mg}$ per $\mathrm{g}$ (weight) of DFAIII or vehicle (control) using a plastic feeding tube every day at the age of 17 weeks old until killed.

Bacterial 16S rRNA amplicon sequencing and analysis. Bacterial genomic DNA was isolated from faeces using a QIAamp DNA Stool mini kit (QIAGEN), and $100 \mathrm{ng}$ of DNA was used for PCR for V1-V4 hyper variable regions of the 16S rRNA gene. Twenty five cycles of amplification was performed with universal $16 \mathrm{~S}$ rRNA primers 27F 5'-AGAGTTTGATCCTGGCTCAG-3' and 519R 5'GWATTACCGCGGCKGCTG-3' with 10-bp barcode tags using KOD Fx plus DNA polymerase (TOYOBO). All amplicons were sequenced on a 454 Genome Sequencer FLX Titanium platform (Roche Diagnostics and Beckman Coulter Genomics). Quality filter-passed sequence reads were obtained by removing reads that had no both primer sequences, had less than $500 \mathrm{bp}$ in length, had the average quality value $(\mathrm{QV})<25$, or were possible chimaeric. Of the filter-passed reads, more than 2,500 sequence reads trimming off both primer sequences for each sample were used and subjected to OTU analysis with the cutoff similarity of $97 \%$ identity using QIIME software. Representative sequences from each OTU were blasted to the database in Ribosomal Database Project (RDP) and aligned. The obtained OTU sequences were grouped at class level $\mathrm{l}^{34,35}$ and were subjected to phylogenetic analysis using MEGA software as described previously ${ }^{35}$.

Determination of the copy number of faecal bacteria. The copy number of faecal bacteria was calculated from the standard curve of known bacterial copy number by quantitative real-time PCR of $16 \mathrm{~S}$ rRNA gene using 341f, $5^{\prime}$ -

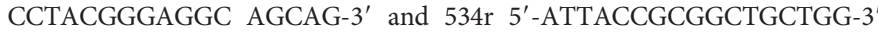
primers as described previously ${ }^{36}$

Measurement of serum ALT and AST. The levels of serum alanine aminotransferase (ALT) and aspartate aminotransferase (AST) were measured using kits from WAKO Pure Chemical Industries, Ltd.

Measurement of serum deoxycholic acid. The metabolomic analysis of mice serum were performed by liquid chromatograph mass spectrometry(LC-MS) in Human Metabolome Technologies Inc. Japan as previously described ${ }^{37}$. The amount of serum DCA was measured by gas chromatograph mass spectrometry (GC-MS) in the Bile Acid Institute of Junshin Clinic, Japan as described ${ }^{38}$.

Human subjects. Informed consent was obtained from all patients according to the protocol approved by the ethics committee of the Japanese Foundation for Cancer Research (JFCR).

Statistical analysis. Data were analysed by unpaired $t$-test with Welch correction (two-side) or Mann-Whitney test (two-side). P-values less than 0.05 were considered significant.

Cell culture. Murine primary HSCs were isolated as previously described ${ }^{18,39}$, and were cultured in Dulbecco's modified Eagle's medium supplemented with $10 \%$ fetal bovine serum in $3 \% \mathrm{O}_{2}$ and $5 \% \mathrm{CO}_{2}$ condition. Human primary HSCs were purchased from Health Science Research Resources Bank and were grown in Dulbecco's modified Eagle's medium supplemented with 10\% fetal bovine serum in $3 \% \mathrm{O}_{2}$ and $5 \% \mathrm{CO}_{2}$ condition.

$\boldsymbol{H}$-ras sequencing. Total RNA was prepared from HCCs and HSCs isolated from tumour regions using TRIzol reagent (Invitrogen). RNA was converted to cDNA by using oligo (dT) primer and a 330-bp PCR fragment containing exon 2 of $\mathrm{H}$-ras gene was amplified with $5^{\prime}$-TGGGGCAGGAGCTCCTGGAT-3' and 5'-GAA GGACTTGGTGTTGTTGA-3' primers. PCR fragments were sub-cloned using Target Clone Plus system (TOYOBO) and were sequenced by using DyeTerminator and Big-Dye cycle sequencing system (Applied Biosystems) as described previously ${ }^{33}$. 
31. Horai, R. et al. Production of mice deficient in genes for interleukin (IL)-1 $\alpha$, IL-1 $\beta$, IL- $1 \alpha / \beta$, and IL-1 receptor antagonist shows that IL- $1 \beta$ is crucial in turpentineinduced fever development and glucocorticoid secretion. J. Exp. Med. 187, 1463-1475 (1998).

32. Reagan-Shaw, S., Nihal, M. \& Ahmad, N. Dose translation from animal to human studies revisited. FASEB J. 22, 659-661 (2008).

33. Yamakoshi, K. et al. Real-time in vivo imaging of $\mathrm{p} 16^{\text {Ink4a }}$ reveals cross talk with p53. J. Cell Biol. 186, 393-407 (2009).

34. Collins, M. D. et al. The phylogeny of the genus Clostridium: proposal of five new general and eleven new species combinations. Int. J. Syst. Bacteriol. 44, 812-826 (1994).
35. Atarashi, K. et al. Induction of colonic regulatory T cells by indigenous Clostridium species. Science 331, 337-341 (2011).

36. Song, Y., Liu, C. \& Finegold, S. M. Real-time PCR quantitation of clostridia in feces of autistic children. Appl. Environ. Microbiol. 70, 6459-6465 (2004).

37. Ooga, T. et al. Metabolomic anatomy of an animal model revealing homeostatic imbalances in dyslipidaemia. Mol. Biosyst. 7, 1217-1223 (2011).

38. Muto, A. et al. Detection of $\Delta^{4}$-3-oxo-steroid $5 \beta$-reductase deficiency by LC-ESIMS/MS measurement of urinary bile acids. J. Chromatogr. B 900, 24-31 (2012).

39. Sekiya, Y. et al. Down-regulation of cyclin E1 expression by microRNA-195 accounts for interferon- $\beta$-induced inhibition of hepatic stellate cell proliferation J. Cell. Physiol. 226, 2535-2542 (2011). 